\title{
BMJ Open Historical database cohort study addressing the clinical patterns prior to idiopathic pulmonary fibrosis (IPF) diagnosis in UK primary care
}

David Thickett, ${ }^{1}$ Jaco Voorham, ${ }^{2}$ Ronan Ryan, ${ }^{2}$ Rupert Jones, ${ }^{3}$ Robina Coker, ${ }^{4}$ Andrew M Wilson, ${ }^{5}$ Sen Yang, ${ }^{2}$ Mandy YL Ow, ${ }^{2}$ Priyanka Raju, ${ }^{2}$ Isha Chaudhry, ${ }^{2}$ Antony Hardjojo, ${ }^{2}$ Victoria Carter, ${ }^{2}$ David B Price (D) ${ }^{2,6}$

To cite: Thickett D, Voorham J, Ryan $\mathrm{R}$, et al. Historical database cohort study addressing the clinical patterns prior to idiopathic pulmonary fibrosis (IPF) diagnosis in UK primary care. BMJ Open 2020;10:e034428. doi:10.1136/ bmjopen-2019-034428

- Prepublication history and additional material for this paper are available online. To view these files, please visit the journal online (http://dx.doi. org/10.1136/bmjopen-2019034428).

Received 19 September 2019 Revised 08 January 2020 Accepted 06 March 2020
Check for updates

\section{(c) Author(s) (or their} employer(s)) 2020. Re-use permitted under CC BY-NC. No commercial re-use. See rights and permissions. Published by BMJ.

For numbered affiliations see end of article.

Correspondence to

Dr David B Price; dprice@opri.sg

\section{ABSTRACT}

Objective To explore the clinical pathways, including signs and symptoms, and symptom progression patterns preceding idiopathic pulmonary fibrosis (IPF) diagnosis. Design and setting A historical cohort study was conducted using primary care patient records from the Optimum Patient Care Research Database.

Participants Patients included were at least 30 years, had IPF diagnosis, identified via clinical-coding and freetext records and had a consultation with a chest specialist prior to IPF diagnosis.

Outcome measures The signs and symptoms in the year prior to IPF diagnosis from clinical codes and freetext in primary care electronic records included: cough, dyspnoea, dry cough, weight loss, fatigue/malaise, loss of appetite, crackles and clubbed fingers. The time course of presentations of clinical features and investigations in the years prior to IPF diagnosis were mapped.

Results Within 462 patients identified, the majority (77.9\%) had a respiratory consultation within 365 days prior to the chest specialist visit preceding the IPF diagnosis recorded in their primary care records. The most common symptoms recorded in the 1 year prior to IPF diagnosis were dyspnoea (48.7\%) and cough (40.9\%); other signs and symptoms were rarely recorded $(<5 \%)$. The majority of patients with cough (58.0\%) and dyspnoea (55.0\%) in the 1 year before IPF diagnosis had multiple recordings of the respective symptoms. Both cough and dyspnoea were recorded in $23.4 \%$ of patients in the year prior to diagnosis. Consultation rates for cough, dyspnoea and both, but not other signs or symptoms, began to increase 4 to 5 years prior diagnosis, with the sharpest increase in the last year. Cough and dyspnoea were often preceded by a reduction in measured weight over 5 years leading to IPF diagnosis.

Conclusion Prolonged cough and/or progressive dyspnoea, especially if accompanied with weight loss, should signal for a referral to specialist assessment at the earliest opportunity.

\section{INTRODUCTION}

Idiopathic pulmonary fibrosis (IPF) is the most common and most lethal of the idiopathic interstitial pneumonias, estimated
Strengths and limitations of this study

- This was the first study to map clinical progression patterns in the years leading to idiopathic pulmonary fibrosis (IPF) diagnosis.

- We used a large primary care database with data available up to 7 years prior to IPF diagnosis and records of patients' secondary care usage.

- We developed a list of relevant clinical features via initial review of patient records, allowing for identification of less common clinical features which may be used to find patterns of symptoms to potentially aid IPF diagnosis.

- A very specific definition of IPF was used in this study requiring specialist consultation and diagnosis of IPF in primary care after the consultation. This may result in the exclusion of cases where there was no primary care record indicating specialist consultation.

- Coding limitations of a database that was designed for clinical practice rather than research, are a weakness. However, we used both symptom coding and free-text data to identify IPF, and clinical features indicative of IPF, increasing detection sensitivity.

to affect 14 to 43 per 100000 population, typically over the age of $50 .^{1-3}$ Patients with IPF have a very poor prognosis, with median years of survival of only 2.5 to 3.5 years and 5 -year survival rate as low as $20 \% .{ }^{45}$ In the $\mathrm{UK}$, the mortality rate for IPF was reported to be 5.10 per 100000 person-years in 2005 to 2008 , increasing by around $5 \%$ per year since $1968,{ }^{6}$ thus signifying a significant and growing healthcare concern.

Diagnosing IPF in primary care is challenging due to the non-specific features in the early stages of the disease, ${ }^{7}$ and IPF is seldom seen by physicians in primary care. ${ }^{8}$ In secondary care, a confident diagnosis requires expertise from a multidisciplinary team of pulmonologists, radiologists and 
pathologists. $^{9} 10$ Identification of early predictors and clinical patterns leading to subsequent IPF diagnosis may help primary care physicians identify potential IPF patients for further examination and guide appropriate referral to specialist respiratory services.

Treatment of IPF had previously been limited to symptom management and palliation, but two antifibrotic drugs, pirfenidone and nintedanib, ${ }^{11}{ }^{12}$ have recently become available. Both treatments may improve IPF disease outcomes ${ }^{13-16}$ and are recommended by the UK National Institute for Health and Care Excellence $e^{17} 18$ and by the American Thoracic Society (ATS) / European Respiratory Society (ERS) / Japanese Respiratory Society (JRS)/ Latin American Thoracic Association (LATA) clinical practice guideline. ${ }^{19}$ To obtain optimal benefit from these novel treatment agents, an early diagnosis is required.

While efforts have been made to study the symptom pathways leading to IPF diagnosis, identifying IPF patients using diagnosis codes alone may erroneously include patients with other conditions. ${ }^{20}$ Thus, this study was conducted to characterise the pattern of signs, symptoms and other clinical predictors preceding IPF diagnosis using a real-life population of patients and more definitive selection criteria for IPF.

\section{METHODS}

\section{Study design}

This was a real-life historical cohort study using electronic medical records from the Optimum Patient Care Research Database (OPCRD), a clinical research database containing records of approximately 7 million patients from over 700 primary care centres across the UK (http://opcrd.co.uk/) ${ }^{21}$ with linked patient-completed asthma questionnaire. Asthma outcome measures within the OPCRD have been validated against patient-reported outcomes and treatment response. ${ }^{22}$ The study was conducted according to the quality standards suggested for observational studies, ${ }^{23}$ including the use of an a priori analysis plan, study registration with a commitment to publish and a well-maintained and monitored study database.

\section{Case definition and inclusion criteria}

The inclusion criteria for this study were: between 30 to 100 years of age at diagnosis date, diagnosis date between 2010 and $1^{\text {st }}$ May 2017, and the diagnosis date must have occurred at least 1 year after registration at a participating general practice (GP). Patients were excluded if they had a code for sarcoidosis, allergic alveolitis, pneumoconiosis, asbestosis or other causes of pulmonary fibrosis. The diagnosis date was defined as the date of the first IPF diagnosis or prescription for IPF-specific pharmacotherapy (pirfenidone or nintedanib). The primary analysis population includes patients who had a code for a consultation with a chest specialist prior to the IPF diagnosis. Selection of patients for analyses is further detailed in the online supplementary data.

\section{Outcome assessments}

The primary outcome of this study was the presence of signs and symptoms in the 1 year prior to IPF diagnosis. The secondary outcomes of this study were: (1) the consultation rates for the signs and symptoms up to 7 years prior to diagnosis, (2) the proportion of patients with respiratory consultations and respiratory tests conducted within 90 and 365 days prior to the chest specialist consultation preceding IPF diagnosis and (3) the relationship between the signs and symptoms.

Respiratory consultation was identified based on the presence of Read codes for the following clinical features: chest/respiratory infection, chest symptom, clubbed fingers, cough, crackles, dyspnoea or sputum. Respiratory tests were identified via Read codes for chest X-ray, chest CT scan, lung function test and chest examination.

The Read codes and free-text terms for signs, symptoms and clinical features investigated in this study were selected via an initial round of manual review of patient records. The methods for the selection and the list of Read codes and free-text terms are elaborated in the online supplementary data.

\section{Statistical analysis}

For all data handling, statistical analyses and figures Stata/ SE V.14.2 and Stata/MP/6 V.15.1 were used. Descriptive statistics were used for baseline characteristics. Continuous variables were summarised as mean (SD) or median (IQR) of non-missing observations while categorical variables were presented as proportions of non-missing observations.

Prevalence of signs and symptoms identified via both Read codes and free-text in the 1 year prior to IPF diagnosis were presented as number (\%). Descriptive statistics were also produced for the number of patients with respiratory consultations and tests conducted within 90 days and within 365 days prior to the chest specialist consultation preceding the diagnosis date.

Principal component analysis with rotation was utilised to visualise the prevalence and co-occurrence of codes for signs, symptoms and other clinical features 90 and 365 days prior to IPF diagnosis in a network plot. All signs and symptoms co-occurrence patterns in the year prior diagnosis were also presented as a table.

Consultation rates for signs and symptoms from up to 7 years prior to IPF diagnosis were generated and expressed as rates per 100 patient-years with 95\% CIs. For each year, only patients who had a complete year of available data contributed to the frequency statistics. The number of patients with at least a certain recording frequency (recording dates/year, in the period of up to 12 years before IPF diagnosis) was also tabulated. Data are shown for, on average, once a year, once every 2, 3 and 4 years. 
Time-course of signs, symptoms and weight measurement of individual patients was plotted to visualise the disease progression patterns up to 12 years leading to IPF diagnosis. Visual evaluation was used to quantify the number of patients with each symptom progression pattern.

Descriptive analysis for the mean (SD) time in years between the first recorded cough and dyspnoea until the diagnosis of IPF was conducted for patients with at least 1 year medical record history before first symptom available and had their symptoms occurring before the IPF diagnosis. The cumulative probability of IPF diagnosis every year since the first symptoms is presented in the form of life tables and Kaplan-Meier plots.

In a sensitivity analysis, all analyses were repeated in an overall IPF patients group consisting of patients with Read codes or free-text mention for IPF diagnosis and/ or medication (detailed in the online supplementary data). For this group, the proportions of patients with respiratory consultations and tests were analysed prior to the date of IPF diagnosis or chest consultation (whichever came first). The study protocol was registered with the European Network of Centres for Pharmacoepidemiology and Pharmacovigilance (ENCePP, registration number EUPAS20994). ${ }^{24}$

\section{Patient and public involvement}

This study does not involve patients. The public was not invited in the design of the study nor the writing of this document.

\section{RESULTS}

\section{Patients}

The patient selection flow is depicted in figure 1. A total of 1166 patients were identified to have IPF diagnosis or IPF-related medication following inclusion and exclusion

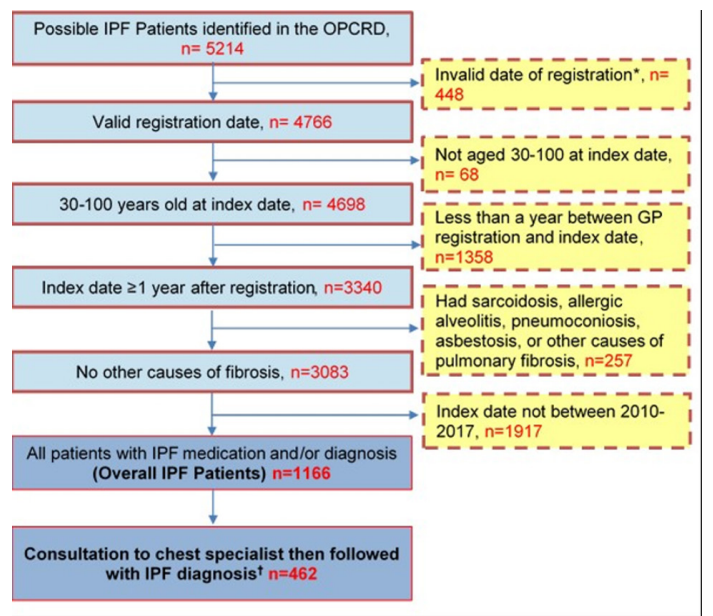

Figure 1 Flowchart of patient selection. ${ }^{\dagger}$ Code for a consultation to a chest specialist followed by an IPF diagnosis (code for consultation allowed until up to 60 days after diagnosis). *Patients with registration date before date of birth. GP,general practice; IPF, idiopathicpulmonary fibrosis; OPCRD, Optimum Patient Care Research Database.
Table 1 Baseline demographic patient characteristics and procedures $(n=462)$

\begin{tabular}{ll}
\hline Variable & Frequency* \\
\hline Age (years) & \\
\hline Mean (SD) & $74.6(9.6)$ \\
\hline Median (IQR) & $75.0(69.0$ to 81.0$)$ \\
Male gender & $272(58.9)$ \\
\hline BMI & \\
\hline $\mathrm{n}$ (\% non-missing) & $442(95.7)$ \\
\hline$<18.5$ & $10(2.3)$ \\
\hline 18.5 to <25 & $137(31.0)$ \\
\hline 25 to <30 & $163(39.8)$ \\
\hline$\geq 30$ & $132(29.9)$ \\
\hline Smoking status & \\
\hline $\mathrm{n}$ (\% non-missing) & $438(94.8)$ \\
\hline Current smoker & $43(9.8)$ \\
\hline Ex-smoker & $236(53.9)$ \\
\hline Never smoked & $159(36.3)$ \\
\hline Asthma diagnosis $\dagger$ & $24(5.2)$ \\
\hline COPD diagnosis $†$ & $19(4.1)$ \\
\hline Respiratory tract cancer & $0(0.0)$ \\
\hline (excluding cancer) & $48(19.0)$ \\
\hline Lung function test conducted & $160(34.6)$ \\
\hline days prior diagnosis & \\
\hline
\end{tabular}

${ }^{*}$ Numbers are presented as $\mathrm{n}(\%)$ unless specified.

†First diagnostic code recorded ever prior and up to diagnosis date.

$\ddagger$ Listed in the online supplementary data.

$\mathrm{BMI}$, body mass index; COPD, chronic obstructive pulmonary disease.

criteria (overall IPF patient group). Of these, 462 patients had a consultation with a chest specialist prior to their IPF diagnosis (primary analysis group).

Of the 462 patients, $58.9 \%$ were male with a mean age at the time of diagnosis was 75 years (table 1 ). The majority $(63.7 \%)$ of patients had a history of smoking, with $9.8 \%$ being current smokers. A total of 88 (19.0\%) patients had spirometry recorded within 90 days and 160 (36.4\%) within 365 days prior to IPF diagnosis. Baseline demographics for the overall IPF patient group $(n=1166)$ were similar (online supplementary table E1).

\section{Primary outcome: signs and symptoms in the 1 year prior to IPF diagnosis}

Of the investigated signs and symptoms, cough and dyspnoea were the most common symptoms recorded in the 1 year prior to IPF diagnosis: cough in 189 (40.9\%) and dyspnoea in $225(48.7 \%)$ patients (table 2). The majority of the patients with cough $(58.0 \%)$ and dyspnoea $(55.0 \%)$ had more than one recording of the respective 


\begin{tabular}{|c|c|}
\hline Signs and symptom & $\mathbf{N}(\%)$ \\
\hline Dyspnoea & $225(48.7)$ \\
\hline Cough* & 189 (40.9) \\
\hline Fatigue or malaise & $22(4.8)$ \\
\hline Weight loss & $19(4.1)$ \\
\hline Crackles & $14(3.0)$ \\
\hline Loss of appetite & $13(2.8)$ \\
\hline Clubbed fingers & $2(0.4)$ \\
\hline Symptom combinations & n (\%) \\
\hline Cough and dyspnoea & $108(23.4)$ \\
\hline Dyspnoea without cough & $117(25.3)$ \\
\hline Cough without dyspnoea & $81(17.5)$ \\
\hline
\end{tabular}

*Of which 25 were 'dry cough'.

IPF, idiopathic pulmonary fibrosis.

symptoms in the 1-year period prior diagnosis. Other signs and symptoms were rarely observed as the first recorded signs and symptoms $(<5 \%)$. Cough and dyspnoea co-occurred on $23.4 \%$ of the patients. The expected rate for both symptoms to co-occur, based on the individual rates, was $20.0 \%$ (95\% CI $18.1 \%$ to $21.9 \%$ ). This was not significantly different from the observed co-occurrence rate (23.4\% (95\% CI $21.4 \%$ to $25.4 \%$ )), suggesting both symptoms occurred independently of each other.

Detailed results including signs and symptoms identified via Read codes only, as well as signs and symptoms in the overall IPF patients, are presented in online supplementary table E2. Compared with using Read codes only, the addition of free-text data increased the number of patients with cough by $8.6 \%$ and dyspnoea by $13.6 \%$.

\section{Secondary outcome: history of respiratory consultation and} respiratory test prior to chest specialist consultation

Around three-quarters of (77.9\%) patients had a respiratory primary care consultation recorded in the 365 days

\begin{tabular}{ll}
\hline $\begin{array}{l}\text { Table } 3 \\
\text { chest specialist consultation prior to IPF diagnosis* }\end{array}$ \\
\hline $\begin{array}{l}\text { Respiratory consultation } \dagger \\
\text { Within } 90 \text { days }\end{array}$ \\
Within 365 days & $360(77.9)$ \\
\hline Respiratory tests conducted & \\
\hline Within 90 days & $176(38.1)$ \\
\hline Within 365 days & $283(61.3)$ \\
\hline
\end{tabular}

${ }^{\star}$ Frequency expressed as $\mathrm{n}(\%)$.

†Codes for chest/respiratory infection, chest symptoms, clubbed fingers, cough, crackles, dyspnoea, or sputum or wheeze. $\ddagger$ Codes for chest X-ray, chest CT scan, lung function test and chest examination.

IPF, idiopathic pulmonary fibrosis. before their chest specialist consultation (table 3). Respiratory tests were recorded in $61.3 \%$ patients in the 365 days prior to the specialist consultation, and more than a third of that $(38.1 \%)$ was within a 90 days period. Prevalence of the components of respiratory consultation and respiratory tests are presented in online supplementary table E3

The proportions of overall IPF patients with respiratory consultations and tests are presented in online supplementary table E4.

\section{Secondary outcome: relationship between signs and symptoms}

The network plots in figure 2 depicts the relationship between signs and symptoms recorded in the 90 days and in the 365 days prior to IPF diagnosis. Strong associations were observed between cough and dyspnoea, and among weight loss, loss of appetite and fatigue/malaise. These two clusters of symptoms were more pronounced in the 90 days period compared with in the 365 days period. Within the extended list of codes which includes clinical features, chest infection was identified to be associated with the cough and dyspnoea cluster. However, chest symptoms, a category containing unspecified chest symptoms, chest examinations and chest CT and X-rays, were more closely associated with the fatigue/malaise cluster. Similar results were observed within the overall IPF patient population (online supplementary figure E1).

The prevalence of every symptom combination within 1 year prior to IPF diagnosis is shown in table 4 . No symptoms were recorded in $30.7 \%$ of the patients. The most common symptom pattern was dyspnoea alone $(22.9 \%)$, cough with dyspnoea $(16.5 \%)$ and cough alone $(13.9 \%)$. Other symptom combinations were rarely observed $(\leq 2 \%$ patients). Symptom combinations in the overall IPF patients are shown in online supplementary table E5.

The frequencies of codes for signs and symptoms up to 7 years leading to the IPF diagnosis are shown in figure 3. A rapid rise in the consultation rate for cough and dyspnoea occurred in the year prior to IPF diagnosis, but the increase in cough and dyspnoea started 4 to 5 years before diagnosis. A similar pattern was observed for consultation for concurrent cough and dyspnoea. This rise was not observed in the other, less common signs and symptoms. The frequencies of codes for signs and symptoms in the overall IPF group are shown in online supplementary figure E2.

The number of patients with at least a certain average symptom frequency in the period before IPF diagnosis (up to 12 years) are presented in online supplementary table E6.

\section{Symptom progression patterns preceding IPF diagnosis}

Several typical patterns of patient pathways were identified from visual assessment of individual patient timelines; cough tended to precede dyspnoea. Weight loss, observed via weight measurements over time, commonly followed recordings of cough and dyspnoea. 244 of the 
90 days
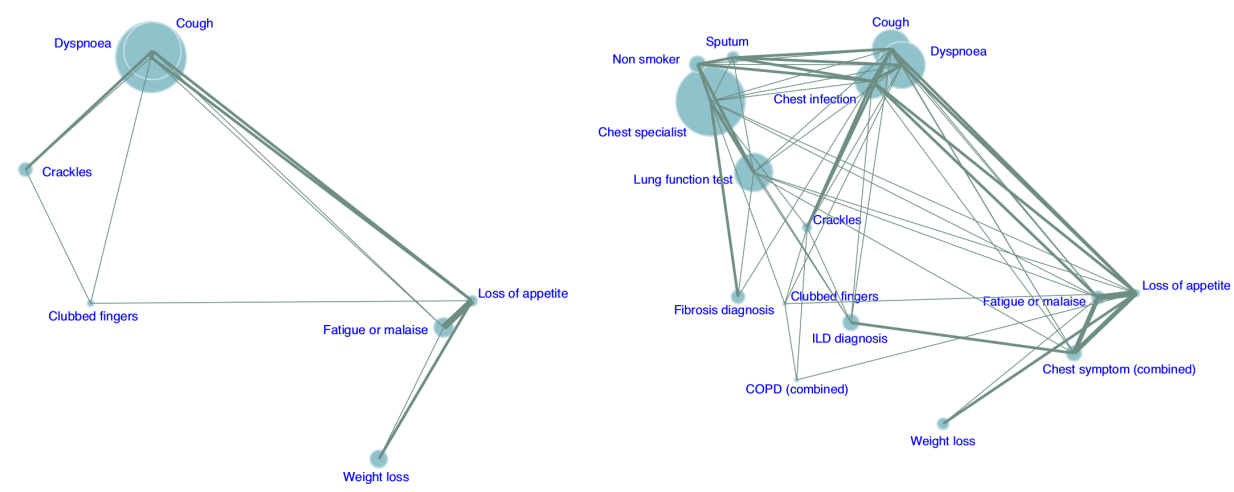

365 days
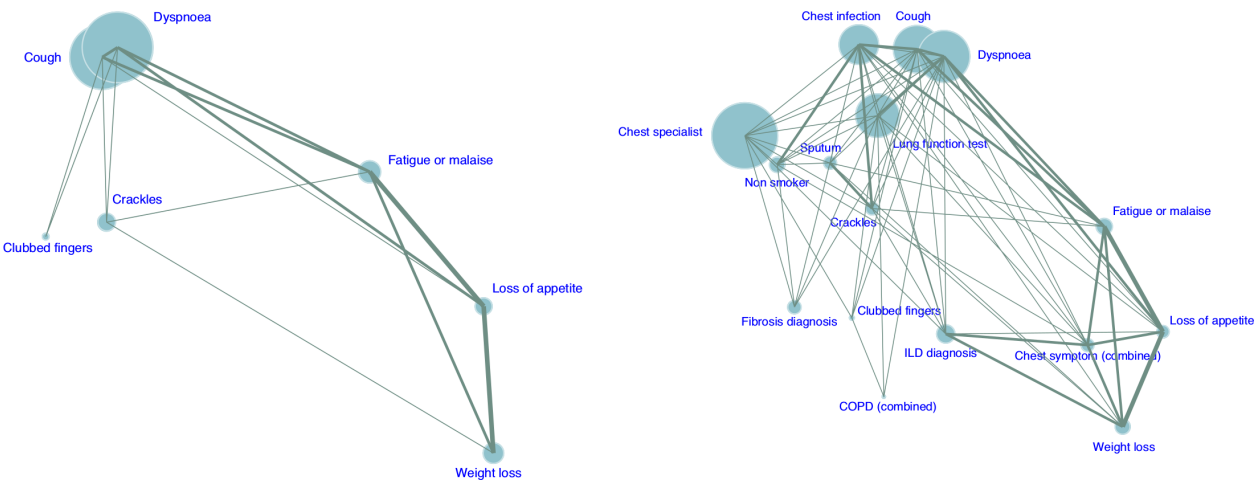

Figure 2 Principal component analysis based network chart for the association between codes within 90 and within 365 days prior to IPF diagnosis. Bubble size indicates prevalence, and thickness of lines indicate the degree of association between signs and symptoms. COPD, chronic obstructive pulmonary disease; ILD, interstitial lung disease; IPF,idiopathic pulmonary fibrosis.

$462(52.8 \%)$ patients $\mathrm{had} \geq 4$ records of weight in the previous 5 years, and of these, $116(47.5 \%)$ recorded weight loss of more than five kilograms. The weight loss often occurred over at least 5 years period (example from two patients in figure 4), however, acute weight loss was also observed (online supplementary figure E3).

Other examples of identified patterns are presented in online supplementary figure E4. A minority, 69 out of $462(14.9 \%)$, received a diagnosis without any symptom codes in the preceding years. Many patients had no clear pattern of respiratory symptoms or weight loss.

\section{Probability of IPF diagnosis from the first recording of symptoms}

Analysis of probability for IPF diagnosis since the first recorded symptom of cough or dyspnoea included 463 patients (322 and 293 patients with cough and dyspnoea, respectively). The mean (SD) time since the first cough was longer (6.3 (5.5) years) compared with since the first dyspnoea (4.3 (4.3) years). Cumulative probability of IPF diagnosis since the first recording of symptoms is illustrated as a life table (online supplementary table E7) and a Kaplan-Meier plot (online supplementary figure E5). These show that $50 \%$ of the patients are diagnosed within 5 years from their first recorded cough, and within 3 years from their first recorded dyspnoea. However, it took, respectively, 13 and 10 years since their first symptoms for $90 \%$ of the patients to receive an IPF diagnosis.

\section{DISCUSSION \\ Main findings}

This is a real-life historical cohort study of IPF patients to identify the symptoms, signs and clinical features recorded before the diagnosis of IPF. Cough and dyspnoea were observed to be the most common recorded symptoms in the 1 year prior to IPF diagnosis, occurring in above $40 \%$ of the patients. Other symptoms, such as weight loss and fatigue, or signs such as crackles and clubbed fingers were rarely observed ( $<5 \%$ patients). The majority of patients $(77.9 \%)$ had a history of at least one primary care respiratory consultation within a year prior to their chest specialist consultation preceding the diagnosis of IPF.

A retrospective review of records from up to 7 years prior to diagnosis demonstrated an increased frequency of codes for cough and dyspnoea starting from 4 to 5 years prior to IPF diagnosis, which further rose sharply nearer to the IPF diagnosis. Thus, repeated consultations for prolonged cough and dyspnoea are likely to be the characteristic symptoms of IPF.

The symptom combination of cough and dyspnoea in the year before IPF diagnosis was observed to be one 


\begin{tabular}{|c|c|c|c|c|c|c|c|c|c|c|}
\hline $\begin{array}{l}\text { Clubbed } \\
\text { fingers }\end{array}$ & Cough & Crackles & $\begin{array}{l}\text { Dry } \\
\text { cough }\end{array}$ & Dyspnoea & $\begin{array}{l}\text { Fatigue or } \\
\text { malaise }\end{array}$ & $\begin{array}{l}\text { Loss of } \\
\text { appetite }\end{array}$ & $\begin{array}{l}\text { Weight } \\
\text { loss }\end{array}$ & Patients & $\%$ & Cumulative $\%$ \\
\hline & & & & & & & & 142 & 30.7 & 30.7 \\
\hline & & & & & & & & 106 & 22.9 & 53.7 \\
\hline & & & & & & & & 76 & 16.5 & 70.1 \\
\hline & & & & & & & & 64 & 13.9 & 84.0 \\
\hline & & & & & & & & 9 & 1.9 & 85.9 \\
\hline & & & & & & & & 9 & 1.9 & 87.9 \\
\hline & & & & & & & & 8 & 1.7 & 89.6 \\
\hline & & & & & & & & 5 & 1.1 & 90.7 \\
\hline & & & & & & & & 4 & 0.9 & 91.6 \\
\hline & & & & & & & & 4 & 0.9 & 92.4 \\
\hline & & & & & & & & 3 & 0.6 & 93.1 \\
\hline & & & & & & & & 3 & 0.6 & 93.7 \\
\hline & & & & & & & & 2 & 0.4 & 94.2 \\
\hline & & & & & & & & 2 & 0.4 & 94.6 \\
\hline & & & & & & & & 2 & 0.4 & 95.0 \\
\hline & & & & & & & & 2 & 0.4 & 95.5 \\
\hline & & & & & & & & 2 & 0.4 & 95.9 \\
\hline & & & & & & & & 1 & 0.2 & 96.1 \\
\hline & & & & & & & & 1 & 0.2 & 96.3 \\
\hline & & & & & & & & 1 & 0.2 & 96.5 \\
\hline & & & & & & & & 1 & 0.2 & 96.8 \\
\hline & & & & & & & & 1 & 0.2 & 97.0 \\
\hline & & & & & & & & 1 & 0.2 & 97.2 \\
\hline & & & & & & & & 1 & 0.2 & 97.4 \\
\hline & & & & & & & & 1 & 0.2 & 97.6 \\
\hline & & & & & & & & 1 & 0.2 & 97.8 \\
\hline & & & & & & & & 1 & 0.2 & 98.1 \\
\hline & & & & & & & & 1 & 0.2 & 98.3 \\
\hline & & & & & & & & 1 & 0.2 & 98.5 \\
\hline & & & & & & & & 1 & 0.2 & 98.7 \\
\hline & & & & & & & & 1 & 0.2 & 98.9 \\
\hline & & & & & & & & 1 & 0.2 & 99.1 \\
\hline & & & & & & & & 1 & 0.2 & 99.4 \\
\hline & & & & & & & & 1 & 0.2 & 99.6 \\
\hline & & & & & & & & 1 & 0.2 & 99.8 \\
\hline & & & & & & & & 1 & 0.2 & 100.0 \\
\hline
\end{tabular}

Secondary outcome: consultation rate in the years leading to IPF diagnosis. IPF, idiopathic pulmonary fibrosis.

of the most common symptom co-occurrence patterns. Using principal component analysis, we have shown the close association between cough and dyspnoea in the preceding 90 and 365 days within patients with IPF. Rates for consultations for both symptoms also increased 4 to 5 years prior diagnosis, similar to the coding of individual symptoms. Thus, prolonged history or presentation of both symptoms is likely to be a 'red flag' to consider IPF and to refer to specialist respiratory services especially when other conditions such as asthma or chronicobstructive pulmonary disease (COPD) have been ruled out. A second cluster between weight loss, loss of appetite and fatigue or malaise was identified. However, these signs and symptoms were too infrequent and unlikely to be helpful for the early identification of IPF in community settings.

Surprisingly, $30.7 \%$ of our patients were not recorded as having any signs or symptoms in the 1 year prior to 
A

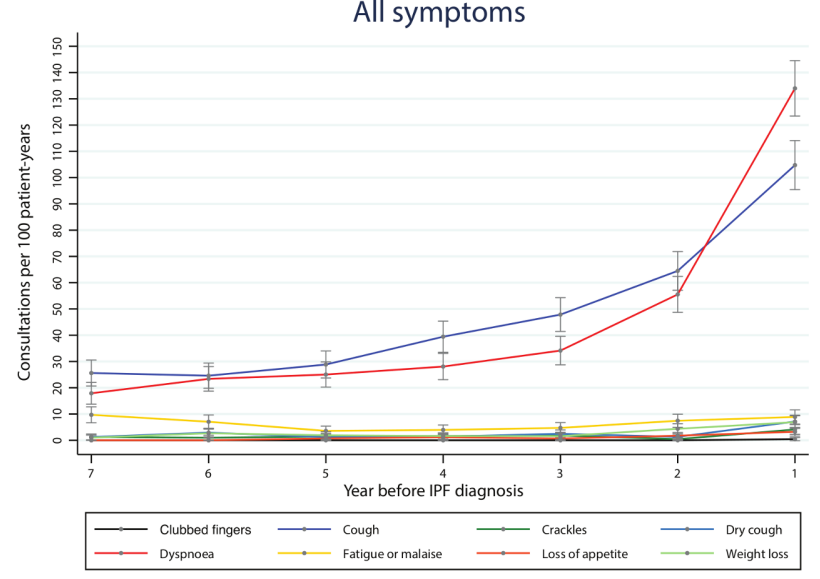

B

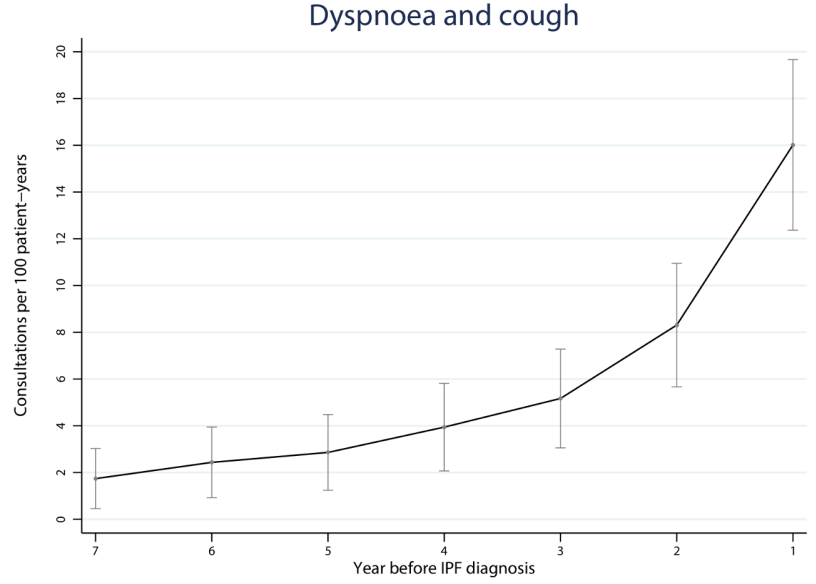

C

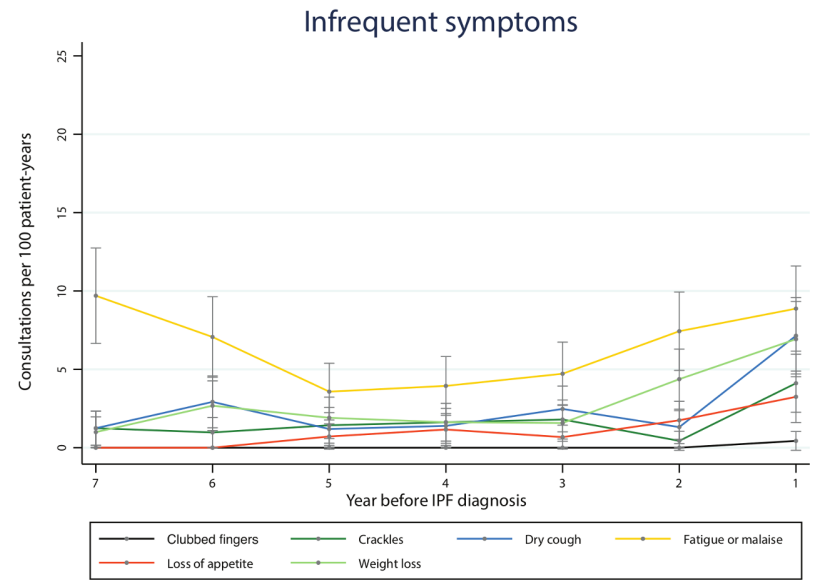

Figure 3 Frequency of codes for signs and symptoms from 7 years prior to IPF diagnosis. (A) All signs and symptoms, (B) co-occurring dyspnoea and cough, $(C)$ excluding cough and dyspnoea. IPF, idiopathic pulmonary fibrosis.

IPF diagnosis (table 4). Analysis of symptom progression patterns also showed that $14.9 \%$ of the patients did not have any symptom codes in the years leading to IPF diagnosis. This may be due to a tendency for primary care physicians to code for the final diagnosis instead of the presenting symptoms during consultations. Alternatively, IPF may be diagnosed coincidentally by investigations for other conditions, such as cardiac CT scanning, without records of respiratory signs and symptoms.

A novelty of the current study is the investigation of symptom progression patterns in the years leading to IPF diagnosis. Cough symptoms tended to precede dyspnoea. However, many of these cough records may have been unrelated to IPF, for example, viral infection. Weight loss was observed to be a common sequel to cough and dyspnoea, occurring over at least 5 years, suggesting the possibility of a disease process preceding the diagnosis by several years. Many patient pathways had no clear pattern of respiratory symptoms or weight loss.

\section{Strengths and limitations}

A strength of this study is the conduct of initial reviews of individual primary care case notes to create the list of Read codes and free-text terms. This enabled less commonly IPF-related signs, symptoms and clinical features to be included within this study, which may have been otherwise missed if a predetermined list of features and codes was used instead.

This study used information from free-text primary care records in addition to diagnostic Read codes. The usage of information from free-text records increased detection sensitivity for signs and symptoms, contributing an additional $8.6 \%$ patients with symptoms of cough and $13.6 \%$ with dyspnoea in the 1 year prior to IPF diagnosis. The recording of respiratory symptoms such as breathlessness has previously been found to occur in free-text well before specific codes are entered in for people with COPD and asthma. ${ }^{25}$

This study used every eligible patient from a primary care records database, with the data availability extending to 7 years prior to the diagnosis of IPF. The real-life design of this study provides high generalisability of the results to primary care patients managed in actual primary care practice. Additionally, the long observation period and the availability of free-text clinical records demonstrate the particular strengths of the OPCRD database for longitudinal studies on rare diseases. Clinical records within participating GPs also contain records of patients' secondary care usage, allowing identification of patients who had previous consultation with a specialist.

In this study, we selected patients who had a consultation with a chest specialist prior to their diagnosis for analysis. This group of patients was selected out of concern that coding for IPF diagnosis may have been entered as a diagnostic query or mistake instead of a definitive diagnosis. Indeed, higher rates of cough and dyspnoea were observed from both Read codes and free-text in patients with specialist consultation prior to IPF diagnosis (online supplementary table E2). Potential bias of GP diagnosis was also addressed by the additional requirement for prior specialist assessment.

The main weakness of this study is our lack of gold standard diagnostic criteria in many patients as we were unable to review the contents of letters from secondary care or hospital records directly. Due to this, it is not possible 

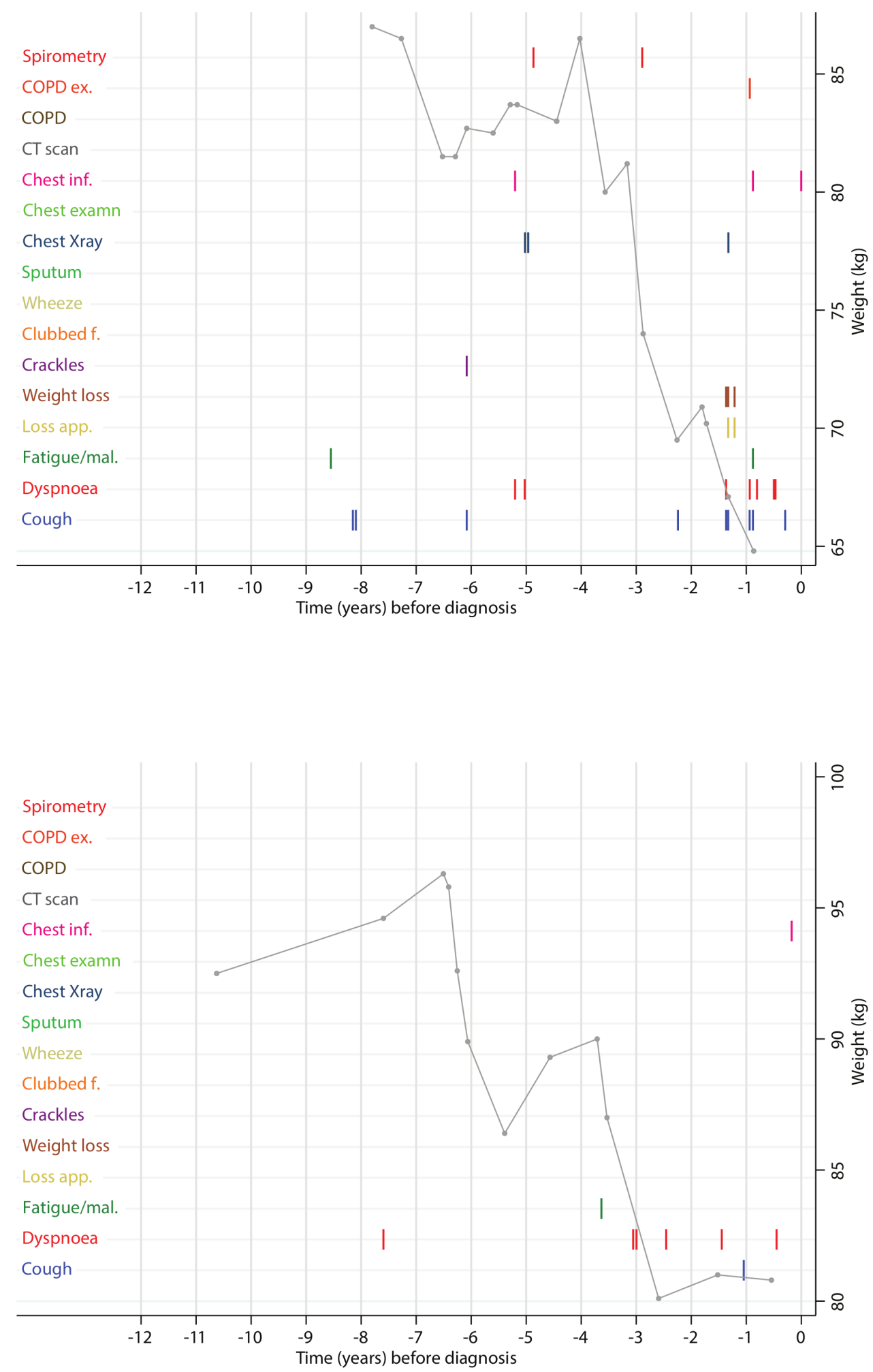

Figure 4 Time course of respiratory symptoms and weight prior to the diagnosis of IPF in two patients showing a long duration of weight loss. COPD,chronic obstructive pulmonary disease; ex., exacerbation; examn, examination; f.,fingers; inf., infection; IPF, idiopathic pulmonary fibrosis; Loss app., lossof appetite; mal., malaise.

to directly confirm that the diagnoses of IPF were made in secondary care, despite the presence of preceding consultation with chest specialists. Our additional selection criterion, requiring prior chest specialist consultation, also resulted in a reduced sample size. Regardless, the pattern of previous respiratory examinations and the outcomes observed seem to support the diagnosis of IPF within the patients. A further weakness is that the primary care records in the OPCRD were initially collected for clinical instead of research purposes, thus the coding of symptoms may be inconsistent. However, the use of freetext searches in this study may mitigate this issue. 
We did not exclude patients with asthma or COPD, which may also cause symptoms of cough and dyspnoea. We felt that asthma and COPD are both common disorders and misdiagnosis of IPF as either symptom are likely. Regardless, the numbers of patients with asthma $(n=24)$ and COPD $(n=19)$ are too small to conduct separate analyses and are unlikely to relevantly change our conclusions. The small number of patients with concomitant COPD is likely due to the unique requirement of the UK primary care system since 2002 in which a diagnosis of COPD requires confirmation by spirometry. Consequentially, the UK may have less misdiagnosis of IPF as COPD than other healthcare systems.

\section{Placing results in the context of published studies}

The observed increase in frequency for cough and/or dyspnoea from 5 years prior IPF diagnosis suggests a delay between recognition of symptoms and diagnosis. Such a delay in the referral and diagnosis of IPF since the initial symptom presentation has been observed in previous studies. ${ }^{26}{ }^{27} \mathrm{~A}$ multicentre cohort study was recently conducted to describe the factors responsible for the delay in IPF diagnosis. ${ }^{28}$ The study reported a mean delay of 2.1 years and that the delay can be mainly attributed to the patients, general practitioners and community hospitals. Due to the importance of early diagnosis of IPF, ${ }^{29}$ there is a need to further understand and rectify the causes for delays in diagnosis and referral.

A previous study investigated the prevalence of symptoms from up to 5 years before IPF diagnosis using another primary care record database in the UK, The Health Improvement Network (THIN) ${ }^{20}$ Similar to our study, the study also reported breathlessness and cough, identified via Read codes, to be the most common symptoms increasing sharply at 1 year prior to IPF diagnosis. However, the study relied solely on Read codes (for idiopathic fibrosing alveolitis (H563.00), Hamman-Rich syndrome (H563.11), cryptogenic fibrosing alveolitis, diffuse pulmonary fibrosis and idiopathic fibrosing alveolitis NOS (not otherwise specified)) to identify patients with IPF. The authors acknowledged that there was a possibility of miscoding leading to the inclusion of patients with other fibrotic lung disorders ${ }^{6}$ and thus labelled cases within their study as 'IPF-clinical syndrome' instead of definitive IPF patients. Our current study confirmed their finding using a more conservative IPF definition (requiring chest specialist consultation prior to the diagnosis) which was expected to be more selective for patients with actual IPF. The current study also extends the previous study by investigating the relationship between the signs and symptoms, demonstrating a common co-occurrence between cough and breathlessness. Furthermore, our study has the additional strength of a longer observational period prior to IPF diagnosis (7 years) and the utilisation of free-text clinical records to identify signs and symptoms which would have been missed by using only Read codes.

\section{CONCLUSIONS}

This is the first study to analyse the presence, association and progression patterns of symptoms and clinical features leading to IPF diagnosis within a more definitive group of patients with IPF, utilising both coded diagnosis and freetext primary care records. Cough and dyspnoea represented the most common symptoms in the 1 year prior to the diagnosis of IPF. Both symptoms were found to be closely associated and were likely to co-occur. While the consultation rates for cough and dyspnoea rose sharply 1 year preceding the diagnosis, the increases in rates were already observable from up to 5 years prior to IPF diagnosis. Lastly, the majority of the patients had records of respiratory consultations and tests conducted prior to their IPF diagnosis.

Taken together, general practitioners should further assess the possibility of IPF in patients who have increasing consultations for prolonged cough and/or progressive dyspnoea, especially if accompanied with weight loss.

Further work will investigate the value of chest X-ray and spirometry in predicting subsequent IPF diagnosis. Linking data within electronic medical records and registries such as BLUETEQ database register of UK specialist drug prescribing ${ }^{30}$ and BTS ILD registries for higher quality research in IPF is also needed to provide deeper insight into the patterns of disease progression identified in this study. Further research comparing the clinical pathway of IPF patients with a control group of patients, as well as investigating whether spirometry led to a timelier referral to specialists may also be warranted.

\section{Author affiliations}

${ }^{1}$ Institute of Inflammation, University of Birmingham, Birmingham, UK

${ }^{2}$ Observational and Pragmatic Research Institute Pte Ltd, Singapore

${ }^{3}$ Peninsula Medical School, University of Plymouth, Plymouth, UK

${ }^{4}$ Imperial College London, London, UK

${ }^{5}$ Respiratory and Airways Group, Norwich Medical School, University of East Anglia, Norwich, UK

${ }^{6}$ Centre of Academic Primary Care, Division of Applied Health Sciences, University of Aberdeen, Aberdeen, UK

Acknowledgements The authors would like to extend their acknowledgement to Dr Dermot Ryan for his clinical and scientific input.

Contributors All authors contributed to the planning, conduct and reporting of this study. DT, RC, AMW, SY, VC and DP conducted the design and planning of this study. $\mathrm{JV}, \mathrm{RR}, \mathrm{RJ}, \mathrm{MO}, \mathrm{PR}, \mathrm{IC}$ and $\mathrm{AH}$ contributed to the data analysis and interpretation. The overall conduct of this study was supervised by DBP. All authors were involved in the drafting of this manuscript and approved the submission of this manuscript.

Funding This study was partly funded by Roche Products Ltd and the Observational and Pragmatic Research Institute (OPRI). Roche was not involved in the preparation, drafting or editing of this manuscript.

Competing interests DT has research contracts with GlaxoSmithKline, Roche and Boehringer Ingelheim. RJ declares grants from AstraZeneca, GlaxoSmithKline and Novartis; and personal fees for consultancy, speakers fees or travel support from AstraZeneca, Boehringer Ingelheim, Chiesi, GlaxoSmithKline, Nutricia, OPRI and Pfizer. DP has board membership with Amgen, AstraZeneca, Boehringer Ingelheim, Chiesi, Circassia, Mylan, Mundipharma, Napp, Novartis, Regeneron Pharmaceuticals, Sanofi Genzyme, Teva Pharmaceuticals; consultancy agreements with Amgen, AstraZeneca, Boehringer Ingelheim, Chiesi, GlaxoSmithKline, Mylan, Mundipharma, Napp, Novartis, Pfizer, Teva Pharmaceuticals, Theravance; grants and unrestricted funding for investigator-initiated studies (conducted through Observational and Pragmatic Research Institute Pte Ltd) from AKL Research and Development Ltd, AstraZeneca, Boehringer Ingelheim, British Lung Foundation, Chiesi, Circassia, Mylan, Mundipharma, Napp, Novartis, Pfizer, 
Regeneron Pharmaceuticals, Respiratory Effectiveness Group, Sanofi Genzyme, Teva Pharmaceuticals, Theravance, UK National Health Service, Zentiva (Sanofi Generics); payment for lectures/speaking engagements from AstraZeneca, Boehringer Ingelheim, Chiesi, Cipla, GlaxoSmithKline, Kyorin, Mylan, Merck, Mundipharma, Novartis, Pfizer, Regeneron Pharmaceuticals, Sanofi Genzyme, Teva Pharmaceuticals; payment for manuscript preparation from Mundipharma, Teva Pharmaceuticals; payment for the development of educational materials from Mundipharma, Novartis; payment for travel/accommodation/meeting expenses from AstraZeneca, Boehringer Ingelheim, Circassia, Mundipharma, Napp, Novartis, Teva Pharmaceuticals; funding for patient enrolment or completion of research from Chiesi, Novartis, Teva Pharmaceuticals, Zentiva (Sanofi Generics); stock/stock options from AKL Research and Development Ltd which produces phytopharmaceuticals; owns $74 \%$ of the social enterprise 0ptimum Patient Care Ltd (Australia and UK) and 74\% of Observational and Pragmatic Research Institute Pte Ltd (Singapore) and is peer reviewer for grant committees of the Efficacy and Mechanism Evaluation programme and Health Technology Assessment. AMW reports investigator-initiated research grant funding from Roche.JV, RR, SY, MO, PR, IC, VC and AJ are employees of Observational and Pragmatic Research Institute Pte Ltd, which has conducted paid research in respiratory disease on behalf of the following organisations in the past 5 years: Anaxys, AstraZeneca, Boehringer Ingelheim, British Lung Foundation, Chiesi, Circassia (formerly Aerocrine), GlaxoSmithKline, Harvey Walsh, Mapi, Morningside Healthcare, Mundipharma, Mylan (formerly Meda), Napp, Novartis, Orion, Plymouth University, Regeneron, Respiratory Effectiveness Group, Roche, Sanofi, Takeda, Teva, University of East Anglia, Zentiva (a Sanofi company).

Patient and public involvement Patients and/or the public were not involved in the design, or conduct, or reporting or dissemination plans of this research.

Patient consent for publication Not required.

Ethics approval The OPCRD is approved by the Health Research Authority of the UK NHS for clinical research use (REC reference: 15/EM/0150), and the protocol for this study was approved by the Anonymised Data Ethics Protocols and Transparency (ADEPT) committee, the independent scientific advisory committee for the OPCRD.

Provenance and peer review Not commissioned; externally peer reviewed.

Data availability statement The data set supporting the conclusions of this article was derived from the 0ptimum Patient Care Research Database (www.opcrd.co. uk). The authors do not have permission to give public access to the study data set; researchers may request access to OPCRD data for their own purposes. Access to OPCRD can be made via the OPCRD website (https://opcrd.co.uk/our-database/ data-requests/) or via the enquiries email info@opcrd.co.uk. The OPCRD has ethical approval from the National Health Service (NHS) Research Authority to hold and process anonymised research data (Research Ethics Committee reference: 15/ EM/0150). This study was approved by the Anonymised Data Ethics Protocols and Transparency (ADEPT) committee- the independent scientific advisory committee for the OPCRD.

Open access This is an open access article distributed in accordance with the Creative Commons Attribution Non Commercial (CC BY-NC 4.0) license, which permits others to distribute, remix, adapt, build upon this work non-commercially, and license their derivative works on different terms, provided the original work is properly cited, appropriate credit is given, any changes made indicated, and the use is non-commercial. See: http://creativecommons.org/licenses/by-nc/4.0/.

ORCID iD

David B Price http://orcid.org/0000-0002-9728-9992

\section{REFERENCES}

1 Spagnolo P, Sverzellati N, Rossi G, et al. Idiopathic pulmonary fibrosis: an update. Ann Med 2015;47:15-27.

2 Raghu G, Chen S-Y, Hou Q, et al. Incidence and prevalence of idiopathic pulmonary fibrosis in US adults 18-64 years old. Eur Respir J 2016;48:179-86.

3 Agabiti N, Porretta MA, Bauleo L, et al. Idiopathic pulmonary fibrosis (IPF) incidence and prevalence in Italy. Sarcoidosis Vasc Diffuse Lung Dis 2014;31:191-7.

4 Ley B, Collard HR, King TE. Clinical course and prediction of survival in idiopathic pulmonary fibrosis. Am J Respir Crit Care Med 2011;183:431-40.
5 Strongman H, Kausar I, Maher TM. Incidence, prevalence, and survival of patients with idiopathic pulmonary fibrosis in the UK. Adv Ther 2018;35:724-36.

6 Navaratnam V, Fleming KM, West J, et al. The rising incidence of idiopathic pulmonary fibrosis in the U.K. Thorax 2011;66:462-7.

7 Zibrak JD, Price D. Interstitial lung disease: raising the index of suspicion in primary care. NPJ Prim Care Respir Med 2014;24:14054.

8 Thickett DR, Kendall C, Spencer LG, et al. Improving care for patients with idiopathic pulmonary fibrosis (IPF) in the UK: a round table discussion. Thorax 2014;69:1136-40.

9 Wells AU. The revised ATS/ERS/JRS/ALAT diagnostic criteria for idiopathic pulmonary fibrosis (IPF)--practical implications. Respir Res 2013;14 Suppl 1:S2.

10 Raghu G, Collard HR, Egan JJ, et al. An official ATS/ERS/JRS/ALAT statement: idiopathic pulmonary fibrosis: evidence-based guidelines for diagnosis and management. Am J Respir Crit Care Med 2011;183:788-824.

11 George PM, Wells AU. Pirfenidone for the treatment of idiopathic pulmonary fibrosis. Expert Rev Clin Pharmacol 2017;10:483-91.

12 Mazzei ME, Richeldi L, Collard HR. Nintedanib in the treatment of idiopathic pulmonary fibrosis. Ther Adv Respir Dis 2015;9:121-9.

13 Meyer KC. Pulmonary fibrosis, part I: epidemiology, pathogenesis, and diagnosis. Expert Rev Respir Med 2017;56:1-17.

14 Meyer KC. Pulmonary fibrosis, part II: state-of-the-art patient management. Expert Rev Respir Med 2017;161:1-16.

15 Vianello A, Molena B, Turato C, et al. Pirfenidone improves the survival of patients with idiopathic pulmonary fibrosis hospitalized for acute exacerbation. Curr Med Res Opin 2019;35:1187-90.

16 Nakano A, Ohkubo H, Fukumitsu K, et al. Remarkable improvement in a patient with idiopathic pulmonary fibrosis after treatment with nintedanib. Intern Med 2018.

17 National Institute for Health and Care Excellence. Nintedanib for treating idiopathic pulmonary fibrosis. Available: https://www.nice. org.uk/guidance/ta379 [Accessed 8 Oct 2018].

18 National Institute for Health and Care Excellence. Pirfenidone for treating idiopathic pulmonary fibrosis. Available: https://www.nice. org.uk/guidance/ta504/chapter/1-Recommendations [Accessed 8 Oct 2018].

19 Raghu G, Rochwerg B, Zhang Y, et al. An official ATS/ERS/JRS/ALAT clinical practice guideline: treatment of idiopathic pulmonary fibrosis. An update of the 2011 clinical practice guideline. Am J Respir Crit Care Med 2015;192:e3-19.

20 Hewson T, McKeever TM, Gibson JE, et al. Timing of onset of symptoms in people with idiopathic pulmonary fibrosis. Thorax 2017 doi:10.1136/thoraxjnl-2017-210177. [Epub ahead of print: 11 Oct 2017].

21 OPCRD. The optimum patient care research database (OPCRD). Available: https://opcrd.co.uk/ [Accessed Jun 2018].

22 Colice G, Chisholm A, Dima AL, et al. Performance of databasederived severe exacerbations and asthma control measures in asthma: responsiveness and predictive utility in a UK primary care database with linked questionnaire data. Pragmat Obs Res 2018;9:29-42.

23 Roche N, Reddel H, Martin R, et al. Quality standards for real-world research. focus on observational database studies of comparative effectiveness. Ann Am Thorac Soc 2014;11 Suppl 2:S99-104.

24 Electronic register of studies, European network of centres for pharmacoepidemiology and pharmacovigilance (ENCePP). Available: http://www.encepp.eu/encepp/studiesDatabase.jsp [Accessed 20 Feb 2015].

25 Hayward RA, Chen Y, Croft P, et al. Presentation of respiratory symptoms prior to diagnosis in general practice: a case-control study examining free text and morbidity codes. BMJ Open 2015;5:e007355.

26 Collard HR, Tino G, Noble PW, et al. Patient experiences with pulmonary fibrosis. Respir Med 2007;101:1350-4.

27 Purokivi M, Hodgson U, Myllärniemi M, et al. Are physicians in primary health care able to recognize pulmonary fibrosis? Eur Clin Respir J 2017;4:1290339.

28 Hoyer N, Prior TS, Bendstrup E, et al. Risk factors for diagnostic delay in idiopathic pulmonary fibrosis. Respir Res 2019;20:103.

29 Cottin V, Richeldi L. Neglected evidence in idiopathic pulmonary fibrosis and the importance of early diagnosis and treatment. Eur Respir Rev 2014;23:106-10.

30 blueteq. Commissioner high cost drugs system. Available: http:// www.blueteq.com/commhcd [Accessed 7 Feb 2019]. 